\title{
High Resolution Medium Energy ion Scattering Analysis for Investigating UltraShallow Junction of Antimony Implanted in Conventional Silicon
}

Talal H. Alzanki ${ }^{1 *}$, Kandil M. Kandil ${ }^{2}$, Chris Jeynes ${ }^{3}$, Brian J. Sealy ${ }^{3}$, Mohammad R. Alenezi ${ }^{1}$, Abdullah Almeshal $^{1}$, Naziha M. Aldukhanand ${ }^{1}$ and Adel Ghoneim ${ }^{2}$

1. Department of Electronic Engineering, Collage of Technological Studies,Public Authority for Applied Education \&Training (PAAET), P.O. BOX 42325 Safat, Kuwait

2. Department of Applied Sciences, Collage of Technological Studies, Public Authority for Applied Education \&Training (PAAET), P.O. BOX 42325 Safat, Kuwait

3. Advanced Technology Institute, School of Electronics and Physical Sciences, University of Surrey, Guildford, GU2 7XH, UK

\begin{abstract}
Forming ultrashallow junction reduces the source / drain channel length, which improves the CMOS (Complementary metal oxide semiconductor) device performance and the speed of the integrated circuits. Antimony ( $\mathrm{Sb}$ ) of low implant energy $2 \mathrm{keV}$ at implant dose $1 \times 10^{15} \mathrm{~cm}^{-2}$ is implanted in conventional silicon wafer (100). Rapid RTA (Thermal annealing technique) is suitable for the as- implanted samples to recover the radiation damaged region and to activate the implanted antimony. This paper investigates the electrical activation of the implanted antimony by estimating its substitutional fraction in the formed ultra junction in silicon wafer by the possibilities of combining the measurements of RBS (Rutherford backscattering spectroscopy) and MEIS (Medium energy ion scattering). It was found that RTA (Rapid thermal annealing) of the as-implanted samples at temperatures $\leq 800{ }^{\circ} \mathrm{C}$, the substitutional antimony fraction and the retained dose also are about $90 \%$ of the nominal dose. However, annealing at temperatures $>800{ }^{\circ} \mathrm{C}$ up to $1,100{ }^{\circ} \mathrm{C}$ antimony precipitate near to the surface and deactivate the electrical conduction by about $50 \%$ with increasing the sheet resistance by about $30 \%$.
\end{abstract}

Key words: Antimony doped Si, ion implantation, rutherford backscattering spectroscopy (RBS), medium energy ion scattering (MEIS), rapid thermal annealing (RTA), shallow junction formation.

\section{Introduction}

Characterising dopant electrical and structural properties following ion implantation and annealing is one of the challenges for modern CMOS (Complementary metal oxide semiconductor) technologies as actually creating highly activated

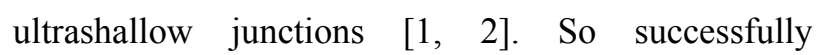
determination of dopant electrical and compositional properties at different depths of the silicon substrate material is of fundamental importance. In a previous

*Corresponding author: Talal H. Alzanki, associate professor, Ph.D., head of the Department of Electronic Engineering, head of academic advising at College of Technological Studies (CTS), public authority for Applied Education and Training (PAAET), Kuwait, research field: nanotechnology. work of Alzanki et al. [3] antimony implantation at 40 $\mathrm{keV}$ and nominal dose $4 \times 10^{14} \mathrm{~cm}^{-2}$ have been characterised for their potential use in n-type shallow junction formation. In that work a comparison is made between DHM (Differential hall effect measurements), which was used to obtain doping carrier profile at depth resolution down to $1 \mathrm{~nm}$, and RBS (Rutherford backscattering) measurements of the atomic profile as a function of annealing temperature. Antimony shows insignificant diffusion for annealing temperature of $800{ }^{\circ} \mathrm{C}$ and below with junction depth of about $60 \mathrm{~nm}$. Electrical activation correlated well with the substitutional fraction of antimony determined by RBS. 
High resolution medium energy ion scattering analysis for quantitative depth profiling of ultra-shallow high - $\mathrm{k}$ layer was investigated by Reading et al. [4]. Their results demonstrate the capability of MEIS (Medium energy ion scattering) to provide high depth resolution, quantitative compositional and structural analysis of high $-\mathrm{k}$ nanofilms. Their work shows interdiffusion, after thermal treatments, of hafnium (Hf) and aluminium (Al) from the cap layer, inserted to modify the metal gate work function. Also, Afrosimov et al. [5] that utilising MEIS for the composition investigation of grapheme oxide films on silicon surface and they conclude that MEIS based diagnostics, when applied to the solving of silicon - grapheme structures synthetic problems, is rather promising and can give much pertinent information. A review of the use of MEIS technique to characterise nanostructures at the surface of a substrate is done by Sortica et al. [6]. They used a method described to simulate MEIS spectra and determine a shape and size distribution of gold nanoparticles adsorbed on a multilayered film of weak polyelectrolyte. They found that the parameters obtained for the nanoparticle from the simulation of experimental MEIS spectra agree quite well with the image obtained by transmission electron microscope TEM (Transmission electron microscope).

This paper is concerned with studying antimony implantation into conventional silicon to form ultra-shallow junction. This junction formation can be achieved by reducing the ion energy of implants. However, low sheet resistance (Rs) is important to allow scaling down (up to nano-scale) of the n-doped junction for CMOS high speed devices. Implantation of antimony at $2 \mathrm{keV}$ and fluence of $1 \times 10^{15} \mathrm{~cm}^{-2}$ is investigated by combining RBS measurements and MEIS at different annealing temperatures $600{ }^{\circ} \mathrm{C}$, $800{ }^{\circ} \mathrm{C}$ and $1,100{ }^{\circ} \mathrm{C}$. RBS is used to measure the implanted antimony dose which is considered to be more accurate (than SIMS profiles) due advantages in particle detection efficiency and less uncertainties concerning energy loss and straggling [7].

\section{Experimental Method}

P-type Silicon (Si) wafers (100), has $100 \mathrm{~mm}$ in diameter and sheet resistance $10-12 \Omega \cdot \mathrm{cm}$, were implanted with antimony $(\mathrm{Sb})$ of low energy $2 \mathrm{keV}$ to a fluence $1 \times 10^{15} \mathrm{~cm}^{-2}$ [8]. The tilt and twist angles of implantation were $7^{\circ}$ and $22^{\circ}$ respectively. The as-implanted wafer then rapidly annealed at temperatures $600{ }^{\circ} \mathrm{C}, 800{ }^{\circ} \mathrm{C}$, and $1,100{ }^{\circ} \mathrm{C}$ for 10 seconds in an inert nitrogen ambient. We investigate and analyze samples before and after annealing using RBS and MEIS data and spectra.

MEIS analysis of the implanted samples was carried out at the Daresbury laboratory facility using the double alignment mode with the $[\overline{111]}$ as the channeling direction, incident angle is $54.7^{\circ}$ [111] and the scattering angle is $70.5^{\circ}$. For Sb implanted $\mathrm{Si}$ sample, $100 \mathrm{keV} \mathrm{He}^{+}$ions and the [111] blocking direction were employed. These conditions formed the best compromise between high depth resolution and sufficient mass separation. The former requires maximizing the path lengths of the $\mathrm{He}^{+}$ions in the sample so that the inelastic energy loss per unit depth inside the $\mathrm{Si}$ is as great as possible. The latter demands maintaining a sufficient energy difference between the $\mathrm{Si}$ and $\mathrm{O}$ and $\mathrm{Si}$ and $\mathrm{Sb}$ peaks in the spectrum, respectively, such that the overlap between deeper lying $\mathrm{Si}$ damage peaks and the $\mathrm{O}$ peak or deeper $\mathrm{Sb}$ and $\mathrm{Si}$ is avoided as reported by J. A. van den Berg [9]. The MEIS analysis and channeling have been used to determine the substitutional fraction of the antimony implant after annealing, measure the retained dose and monitor gross diffusion during annealing. In order to investigate the loss of electrical activity at higher annealing temperatures, MEIS analysis was used to determine the non-substitutional fraction, from which cluster formation may be inferred according to Jeynes et al. [7]. 


\section{Results and Discussion}

A good representation of the relative quantities of different elements in a material can be determined as a function of depth from RBS spectra as shown in Fig. 1. More simply, by directing the beam in a "random" direction in the substrate an accurate estimation of antimony retained dose can be attained. Likewise, by directing the beam through an obvious channel of the crystalline lattice, $(<100>$ of $\mathrm{Si}$ substrate), a spectrum is assembled that contain an information about antimony present in the channel of the silicon. So, an estimation of the substitutional fraction of antimony can be made by looking at relative quantities of random and interstitial fraction by the relation [1 (aligned/random)] [7]. In this case while every substitutional dopant may not necessarily contribute electrically, it is generally accepted that for a dopant atom to act as donor or acceptor it should substitutional in the host lattice [10].

When characterizing the ultrashallow implants (junction) the resolution of RBS becomes a trivial limiting factor because of the relatively deep penetration of the beam. One way to overcome this issue is to combine RBS and MEIS spectroscopy, which is a refinement of RBS with enhanced depth and angle resolution as a result of ${ }^{4} \mathrm{He}^{+}$ion beam having lower energy $100 \mathrm{keV}$.

The description of "random" spectra of $2 \mathrm{keV}$ implanted antimony with a nominal dose $1 \times 10^{15} \mathrm{~cm}^{-2}$ as a function of the annealing temperature is shown in Fig. 2. In this figure, the spectra of the sample annealed at $600{ }^{\circ} \mathrm{C}$ shows nearly complete fit with the as-implanted which means that almost of the implanted antimony participate in the silicon lattice as a substitutional dopant atom. The RBS retained does with the active retained dose (sheet carrier concentration $\mathrm{N}_{\mathrm{s}}=$ electron density $\mathrm{cm}^{-2}$ ) calculated in a previous part of the work from Differential Hall measurements [3] are presented in Table 1. As can be seen in Table 1, the electrically active fraction of antimony dopant, which is related directly to the substitutional fraction of the dopant, is calculated from the relative of Ns with RBS retained dose and also from the relative of Ns with the nominal dose and then finally estimated from MEIS data. The fraction of antimony detected in the channel and therefore non-substitutional can be deduced from the ratio of the "aligned" and "random" antimony counts. This can be

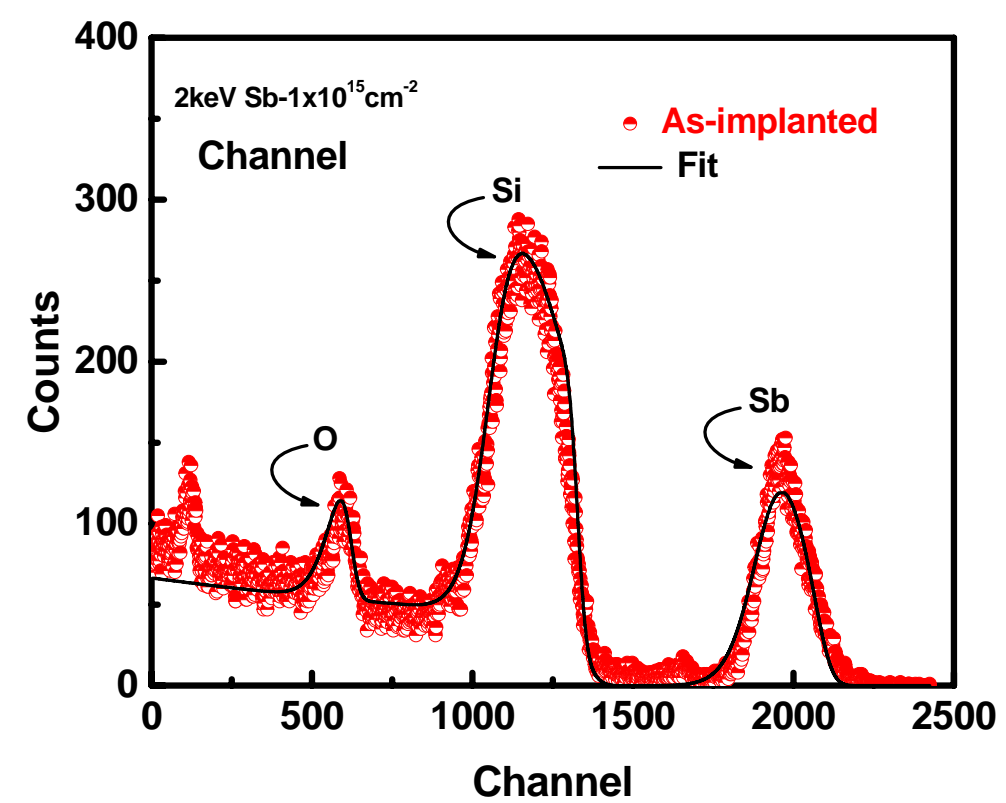

Fig. 1 shows the RBS good fitting of the channeling spectra of as-implanted sample of implant energy $2 \mathrm{keV}$ with a fluence 1 $\times 10^{15} \mathrm{~cm}^{-2}$ at room temperature. This partial spectra are shown for the channeled antimony and the non-channeled surface layers of silicon and oxygen. 


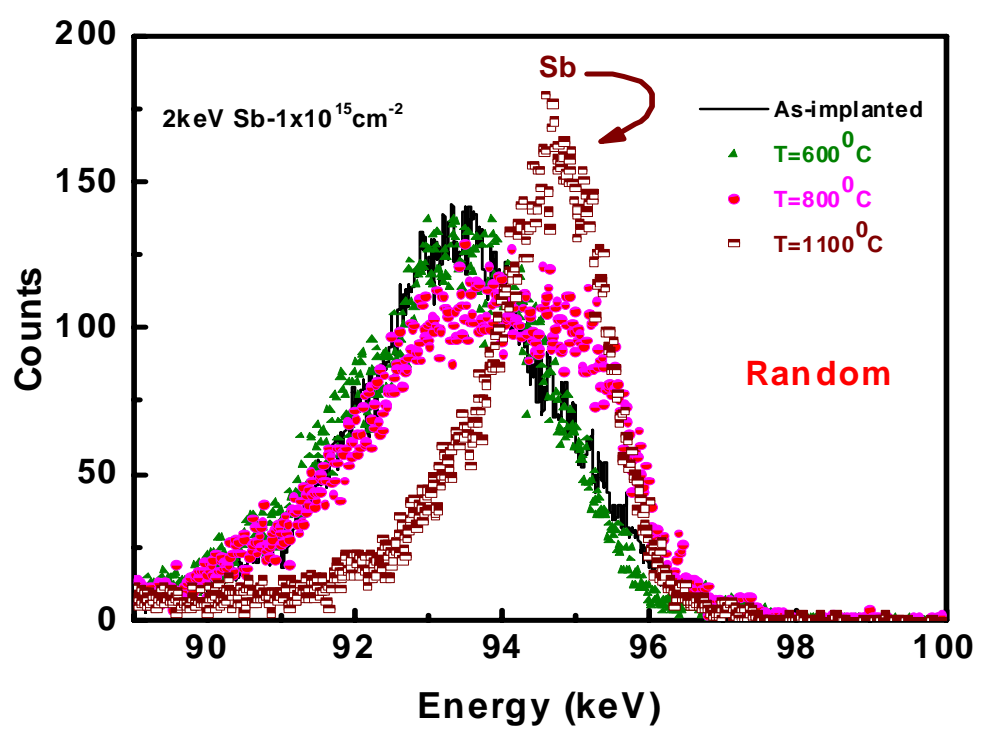

Fig. 2 MEIS random (R) spectra of Si samples implanted with $2 \mathrm{keV} \mathrm{Sb^{+ }}$ at a dose of $1 \times 10^{15} \mathrm{~cm}^{-2}$, the as-implanted spectrum and annealed at $600{ }^{\circ} \mathrm{C}, 800^{\circ} \mathrm{C}$ and $1,100{ }^{\circ} \mathrm{C}$.

Table 1 Retained dose from RBS and Hall measurement with chemical substitutional fraction and sheet resistance $\mathbf{R}_{\mathrm{s}}$.

\begin{tabular}{|c|c|c|c|c|c|c|c|}
\hline $\begin{array}{l}\text { Annealing } \\
\text { temperature } \\
{ }^{\circ} \mathrm{C}\end{array}$ & $\begin{array}{l}\text { Nominal } \\
\text { implant } \\
\text { dose } \\
\mathrm{cm}^{-2} \\
\end{array}$ & $\begin{array}{l}\text { RBS } \\
\text { retained } \\
\text { dose } \\
\mathrm{cm}^{-2} \\
\end{array}$ & $\begin{array}{l}\mathrm{N}_{\mathrm{s}}=\text { Active } \\
\text { retained } \\
\text { dose } \mathrm{cm}^{-2} \\
(\text { Hall }[3])\end{array}$ & $\begin{array}{l}\text { Electrical } \\
\text { activation } \\
\text { fraction \% } \\
\mathrm{N}_{\mathrm{s}} / \text { RBS }\end{array}$ & $\begin{array}{l}\text { Electrical } \\
\text { activation } \\
\text { fraction } \% \\
\mathrm{~N}_{\mathrm{s}} / \text { Nominal } \\
\end{array}$ & $\begin{array}{l}\text { Chemical } \\
\text { substitutional } \\
\text { fraction } \%\end{array}$ & $\begin{array}{l}\mathrm{R}_{\mathrm{s}} \\
\Omega / \square \\
{[3]}\end{array}$ \\
\hline As-Implanted & $1 \times 10^{15}$ & & & & & & \\
\hline $600^{\circ} \mathrm{C}$ & $1 \times 10^{15}$ & $8.1 \times 10^{14}$ & $9.9 \times 10^{13}$ & 12 & $10 \rightarrow 12$ & 10.3 & 1,050 \\
\hline $800^{\circ} \mathrm{C}$ & $1 \times 10^{15}$ & $8.1 \times 10^{14}$ & $7.8 \times 10^{13}$ & 9.6 & $8 \rightarrow 10$ & 8.2 & 1,164 \\
\hline $1,100^{\circ} \mathrm{C}$ & $1 \times 10^{15}$ & $7.9 \times 10^{14}$ & $4.2 \times 10^{13}$ & 4.2 & $4 \rightarrow 6$ & 4.2 & 1,404 \\
\hline
\end{tabular}

converted to a substitutional antimony fraction by the relation [1 - (aligned counts/random counts)]. These values in Table 1 are nearly the same within experimental error. This is reflected also from the random spectra of the sample annealed at $800{ }^{\circ} \mathrm{C}$ as in Fig. 2, where a less peak intensity is observed relative to that of the as- implanted and that annealed at $600{ }^{\circ} \mathrm{C}$ with a shift towards the substrate surface but no in-diffusion. This means antimony starts to segregate towards the surface. For the sample annealed at higher temperature $1,100{ }^{\circ} \mathrm{C}$, the effect of antimony out-movement is more pronounced which suggests that antimony precipitate near the surface, perhaps as a result of oxidation. The spectra can be fitted very well by assuming that there is an oxide layer at the surface for the annealed samples.

Fig. 3 shows the aligned spectra of $2 \mathrm{keV}$ implanted antimony at $1 \times 10^{15} \mathrm{~cm}^{-2}$. This figure shows that a buildup of non-substitutional antimony near the surface as the annealing temperature increased to $800{ }^{\circ} \mathrm{C}$. This behavior is more pronounced at $1,100{ }^{\circ} \mathrm{C}$ as appeared by the strength of the signal and by the shift in its energy position. Thus antimony moves towards the surface where it tends to precipitates and become electrically inactive with increasing the sheet resistance as shown in Table 1. From the counts of the aligned spectra and that of the random one the chemically substitutional antimony fraction is obtained and comparable with the electrically active fraction is included in Table 1 . The results are in good agreement within experimental error. So as shown from this study an ultrashallow junction of silicon doped antimony could be obtained with lowering the annealing temperature $<800{ }^{\circ} \mathrm{C}$ at lower implant does due to high substitutional fraction of antimony [11]. 


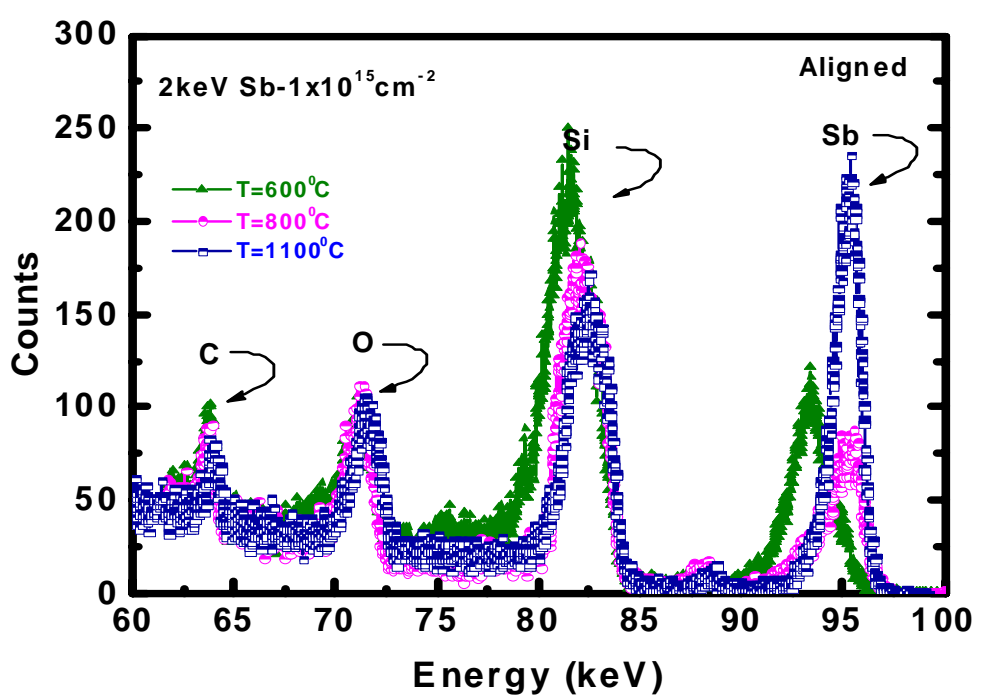

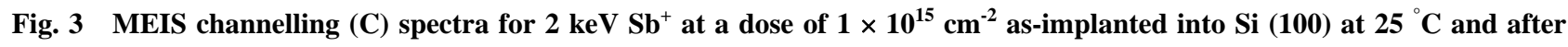
annealing for $10 \mathrm{~s}$ at $600{ }^{\circ} \mathrm{C}, 800{ }^{\circ} \mathrm{C}$ and $1,100{ }^{\circ} \mathrm{C}$. Spectra shows the surface layers $\mathrm{Si}, \mathrm{O}$ and $\mathrm{C}$.

\section{Conclusions}

Combination of RBS measurements and MEIS measurements it can merit reliable results. Information about antimony as a dopant for conventional silicon substrate by different techniques give a good insight about its role in forming ultrashallow junction for CMOS devices. The electrical activation due to the implanted antimony of low energy $2 \mathrm{keV}$ at fluence 1 $\times 10^{15} \mathrm{~cm}^{-2}$ is reduced $50 \%$ by rapid thermal annealing at temperature $>800{ }^{\circ} \mathrm{C}$ which is due to the reduction of substitutional antimony in silicon lattice with an increase of its precipitates near the surface.

The decrease in electrical activation is accompanied by increase in the sheet resistance which is expected due to the increase of non-substitutional and segregate of antimony towards the surface with the increase of the annealing temperature up to $1,100{ }^{\circ} \mathrm{C}$.

\section{Acknowledgements}

The authors thank the Kuwait Government, this work has been supported by the Public Authority of Applied Education and Training (PAAET) of the State of Kuwait (grant TS-15-01). We also thank the University of Surrey Ion Beam Centre and the United Kingdom (UK) Engineering.

\section{References}

[1] International Technology Roadmap for Semiconductors. 2009. “ITRS. Roadmap Report." http://www.itrs.net.

[2] Vandervorst, W. 2007. "USJ Metrology: from 0D to 3D Analysis." International Conference on Frontiers of Characterization and Metrology for Nanoelectronocs. AIP Conf. Proc. 931 (1): 233.

[3] Alzanki, T., Gwilliam, R., Emerson, N., Tabatabaian, Z., Jeynes, C. and Sealy, B. J. 2004. "Concentration Profiles on Antimony Doped Shallow Layers in Silicon." Semi, Sci.\& Technol. 19: 728.

[4] Reading, M. A., Van der Berg, J. A., Zalm, P. C., Armour, D. G., Baily, P. and Noakes, T. C. Q. et al. 2010. "High Resolution Medium Energy Ion Scattering Analysis for the Quantitative Depth Profile for Ultrathin High-k Layer.” J. Vac. Sci. Technol. B 28 (1):C1-C65.

[5] Afrosimov, V. V., Dideykin, A. T., Skharon, V. I., Serenkov, I. T. and Vul, S. P. 2014. "Utilizing of Medium Energy Ion Scattering Spectroscopy for the Composition Investigation of Grapheme Oxide Films on Silicon Surface," Nanosystems: Phys. Chem. Math. 5 (1): 113-6.

[6] Sortica, M. A., Grand, P. L., Machado, G. and Miotti, L. 2009. "Characterization of Nanoparticles through Medium Energy Ion Scattering." J. Appl. Phys. 106: 114320.

[7] Jeynes, C., Barradas, N. B., Marriott, P. K., Boudreault, G., Jenken, M. and Wendler E. 2003. "Elemental Thin Film Depth Profile by Ion Beam Analysis Using Simulated Annealing - A New Tool.” J. Phys. D: Appl. Phys. 36 (7): R97-R126.

[8] Sealy, B. J., Smith, A. .J., Alzank, T., Bennett, N., Li, L. 
and Jeynes, C. et al. 2006. "Shallow Junction in Silicon with Low Thermal Budget Processing." In IEEE Proceedings of the $6^{\text {th }}$ International Workshop on Junction Technology, 10-5.

[9] Van den Berg, J. A., Armour, D. G., Zhang, S., Whelan, S., Ohnos, H. and Wang, T. S. et al. 2002. "Characterization by Medium Energy Ion Scattering of Damage and Dopant Profiles Produced by Ultrashallow B and as Implants into Si at Different Temperature." J. Vac.
Sci. Technol. B 20 (3): 974.

[10] Pichier, P. 2004. "Intrinsic Point Defects, Impurities and Their Diffusion in Silicon.” Birkhauser: Springer, New York.

[11] Alzank, T., Kandi, K. M., Bennett, N., Sealy, P. J., Alenez, M. R. and Almeshal, A. et al. 2014. "Nanotechnology Investigation of Ultrashallow Junctions of Antimony Implants in Conventional Silicon." SOJ Mater. Sci. \& Eng. 2 (1): 1-6. 